\title{
Role of non-coding RNAs in the neuroadaptation to alcoholism and fetal alcohol exposure
}

\author{
Matthew Reilly* \\ Division of Neuroscience and Behavior, National Institute on Alcohol Abuse and Alcoholism, National Institutes of Health, Rockville, MD, USA \\ *Correspondence: reillymt@mail.nih.gov
}

The control of gene expression is a fundamental process in all of biology. Understanding the mechanisms by which genes are turned off and on in a temporally and spatially mediated fashion, represents an area where major scientific advances are likely to occur in the next decade. This is because the control of the timing and location of gene expression is arguably one of the most critical regulation points in determining cellular identity and function and when gene regulation is perturbed disease states can arise. Classic gene expression control mechanisms such as cis-acting elements in gene promoters and trans-acting factors (transcription factor proteins) have been well studied. However, the recent discovery of gene expression regulation mediated by RNA molecules that are transcribed from DNA but do not code for protein, has set into motion a revolution in molecular biology. These novel RNAs are classified broadly as non-coding RNAs (ncRNAs) and include both small (microRNAs or miRNAs) and large classes (long non-coding RNAs or lncRNAs) that function to alter the expression of genes to which they bind and modify chromatin states. Yet, there remains much to be understood about the biology of ncRNAs and how this contributes to susceptibility to disease.

Like all complex traits, alcoholism is influenced by multiple genetic and environmental factors. In addition, there is significant heterogeneity found with alcoholism making it a daunting task to identify the specific genes associated with the disease. Although progress has been made in identifying some of the genetic variants, explaining the entire phenotypic variation associated with alcoholism is far from complete. Most of the recent efforts in understanding the genetic vulnerability to alcoholism have focused on genotype-phenotype associations. For example, in the last several years there has been an explosion in Genome-Wide Association Studies (GWAS), which seek to correlate common genetic variation (represented by Single Nucleotide Polymorphisms or SNPs) with a particular trait or disease. Success of GWAS has varied across different complex traits, where most studies have found that individual SNPs account for a very small proportion of the variance. This is true for GWAS studies of alcoholism and alcohol-related phenotypes, where genome-wide significance has rarely been achieved. There are several prevailing hypotheses that have been put forth to explain the small effect sizes observed with GWAS and other approaches. These include arguments for the contribution of untested rare variants, sample power issues, and "phantom heritability." It is clear, however, that new perspectives for understanding genetic vulnerability to alcoholism are warranted, and the burgeoning area of microRNAs appears to be a fruitful area of investigation. This is because determining the role of microRNAs in disease will offer both mechanistic and potential therapeutic insight.

Gene expression changes after alcohol exposure are well documented. In particular, a vast network of expression changes are found in the brain (and other tissues) following both acute and chronic alcohol exposure. These neuroadaptations are thought to underlie tolerance and dependence on alcohol as well as mediating the toxic effects of alcohol on neurodevelopment. Studies examining the effects of alcohol on microRNA expression are just beginning. Applying this new information on microRNAs to genome-wide studies of gene expression changes after alcohol exposure represents both a significant challenge and tremendous opportunity for unraveling the genetic vulnerability to alcohol dependence. In this special issue of Frontiers in Genetics two important areas relevant to microRNA biology and alcohol are presented. The first article presents recent studies on microRNAs in post-mortem human brain samples from alcoholics compared to non-alcoholics. This is the first study to profile changes in the expression of microRNAs in brain samples from alcoholics. These changes in microRNAs are correlated with previously identified gene expression changes to provide a glimpse into the possible mechanisms by which alcohol neuroadaptation alters the brain. The second article in this special issue examines the effects of fetal alcohol exposure on microRNA expression. This exciting area has important implications for understanding and treating Fetal Alcohol Syndrome and Fetal Alcohol Spectrum Disorders. Collectively, these studies highlight the emerging role of microRNAs in neurobiology in general and alcohol neuroadaptation specifically. Because microRNAs can finetune gene expression in subtle yet critical ways, harnessing these endogenous master regulators holds tremendous promise for the identification of novel drug targets and eventually the development of new therapeutics to treat alcoholism.

Received: 19 March 2012; accepted: 12 April 2012; published online: 15 June 2012. Citation: Reilly M (2012) Role of non-coding RNAs in the neuroadaptation to alcoholism and fetal alcohol exposure. Front. Gene. 3:70. doi: 10.3389/fgene.2012.00070

This article was submitted to Frontiers in Non-Coding RNA, a specialty of Frontiers in Genetics.

Copyright (C) 2012 Reilly. This is an open-access article distributed under the terms of the Creative Commons Attribution Non Commercial License, which permits non-commercial use, distribution, and reproduction in other forums, provided the original authors and source are credited. 